\title{
Genetic Characterization of Pyridine Nucleotide Uptake Mutants of Salmonella typhimurium
}

\author{
By MICHAEL P. SPECTOR, JEAN M. HILL, ELIZABETH A. HOLLEY \\ AND JOHN W. FOSTER* \\ Department of Microbiology, Marshall University School of Medicine, \\ Huntington, WV 25701, USA
}

(Received 25 July 1984; revised 14 November 1984)

\begin{abstract}
Two classes of pyridine nucleotide uptake mutants isolated previously in a strain of Salmonella typhimurium defective in both de novo NAD biosynthesis ( $\mathrm{nad}$ ) and pyridine nucleotide recycling $(p n c A)$ were analysed in terms of their genetic relationship to each other and their roles in the transport of nicotinamide mononucleotide as a precursor to NAD. The first class of uptake mutants, pnuA (99 units), failed to grow on nicotinamide mononucleotide (NMN) as a precursor for NAD. The second class, pnuB, grew on lower than normal levels of NMN and suppressed pnuA mutations. A third class of uptake mutant, pnuC, isolated in a nadB pncA pnuB background, also failed to grow on NMN. Transport studies and enzyme analyses confirmed these strains as defective in NMN uptake. A fourth locus, designated $p n u D$, was found to diminish NMN utilization in a nad pnc $A^{+}$background. Tn 10 insertions near $p n u A, p n u C$ and $p n u D$ were isolated and utilized in mapping studies. $p n u A$ was found to map between $t h r$ and $\operatorname{ser} B$ near $\operatorname{trp} R$. The $p n u C$ locus was cotransducible with $n a d A$ at 17 units while $p n u D$ mapped at approximately 60 units. The biochemical and genetic data suggest that the pnuA and pnuC gene products cooperate in the utilization of $\mathrm{NMN}$ under normal conditions. A pnuB mutant, however, does not require the $p n u A$ gene product for NMN uptake but does rely on the pnuC product. Fusion studies indicate that $p n u C$ is regulated by internal NAD concentrations.
\end{abstract}

\section{INTRODUCTION}

The maintenance of a proper oxidation-reduction balance is of the utmost importance to living cells. NAD and NADP are two major compounds involved with cellular oxidationreduction reactions. Consequently, the synthesis, recycling and regulation of these coenzymes is of great interest.

NAD may be recycled in Salmonella typhimurium via two pathways, known as pyridine nucleotide cycles (PNC) (Foster \& Moat, 1980). PNC IV recycles NAD through a series of four reactions while PNC VI requires six enzyme steps to accomplish this task (Fig. 1). All available evidence indicates that PNC IV is used primarily for the intracellular recycling of NAD while the major role of PNC VI is in the utilization of preformed pyridine derivatives present in the extracellular environment (Foster \& Baskowsky-Foster, 1980).

Cells with an intact PNC VI can efficiently utilize exogenously supplied nicotinamide mononucleotide (NMN), nicotinamide (NAm) and nicotinic acid (NA) at $10^{-5} \mathrm{M}$. However, a cell containing a mutation in $p n c A$, which eliminates all NAm deamidase activity, cannot utilize NAm and requires an NMN concentration of $10^{-4} \mathrm{M}$ or more. NMN can apparently traverse the membrane intact via a system dependent upon the $p n u A$ locus, which maps at 99 units (Foster et al., 1979a, b; Liu et al., 1982). The efficiency of pnuA-dependent NMN transport

\footnotetext{
Abbreviations: PNC, pyridine nucleotide cycles; NMN, nicotinamide mononucleotide; NAm, nicotinamide; NA, nicotinic acid; NAmR, nicotinamide ribonucleoside.
} 


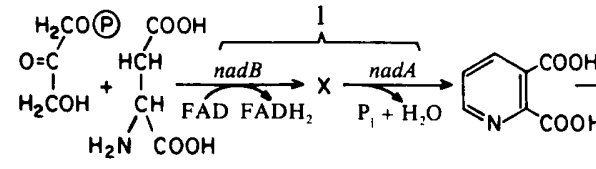

DHAP Aspartate

Quinolinate

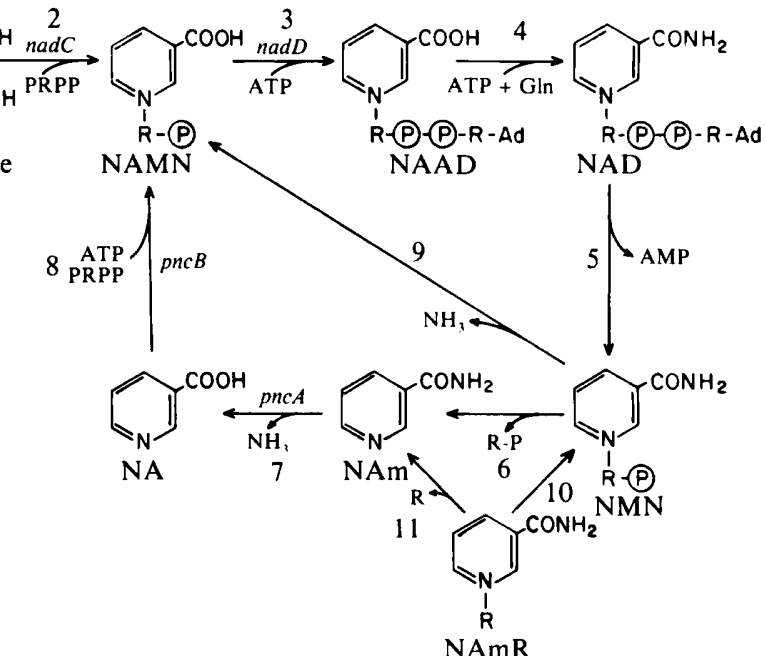

Fig. 1. De novo pathway of NAD biosynthesis and the pyridine nucleotide cycle. The relationship of known genetic markers is shown. The $\mathrm{X}$ in the de novo pathway indicates iminoaspartate. The enzyme designations are as follows: 1 , quinolinic acid synthetase ; 2 , quinolinic acid phosphoribosyltransferase ; 3, nicotinic acid mononucleotide (NAMN) adenylyl transferase; 4, NAD synthetase; 5, NAD pyrophosphatase or DNA ligase; 6, nicotinamide mononucleotide (NMN) glycohydrolase; 7, nicotinamide (NAm) deamidase; 8 , nicotinic acid (NA) phosphoribosyl transferase; 9, NMN deamidase; 10 , nicotinamide ribonucleoside (NAmR) kinase; and 11 , NAmR nucleosidase. DHAP, dihydroxyacetone phosphate; NAAD, nicotinic acid adenine dinucleotide; PRPP, phosphoribosyl pyrophosphate.

appeared to be increased via a secondary mutation in $p n u B$ (Liu et al., 1982). This is shown by the ability of a pnuA $A^{+} p n u B$ mutant to grow on $10^{-5} \mathrm{M}-\mathrm{NMN}$. In the present communication, the relationship between $p п u A$ and $p n u B$ is more clearly defined. In addition, mutations in a third locus, $p n u C$, which suppresses the $p n u B$ phenotype, and a fourth locus, $p n u D$, are characterized.

\section{METHODS}

Bacterial strains and culture conditions. All the bacterial strains used were derivatives of Salmonella typhimurium LT-2; they are listed in Table 1. The minimal E medium of Vogel \& Bonner (1956), supplemented with $0.4 \%$ glucose, was used as a basal medium. Minimal lactose base (MLB) medium was used to test for galactose and lactose utilization (Foster et al., 1979a). Amino acids were supplemented at a final concentration of $40 \mu \mathrm{g} \mathrm{ml}^{-1}$. All $\mathrm{Nad}^{-}$mutants were grown on minimal medium with NA or NAm at a final concentration of $10^{-5} \mathrm{M}, \mathrm{NMN}$ at a final concentration of $10^{-4} \mathrm{M}$ or $10^{-5} \mathrm{M}$ or nicotinamide ribonucleoside (NAmR) at a final concentration of $10^{-4} \mathrm{M}$. All vitamins and intermediates were sterilized by filtration (membrane pore size $0.45 \mu \mathrm{m}$ ). Tetracycline was used at a final concentration of $20 \mu \mathrm{g} \mathrm{ml}^{-1}$ for complex medium and $10 \mu \mathrm{g} \mathrm{ml}^{-1}$ for minimal medium. Ampicillin was used at a final concentration of $30 \mu \mathrm{g} \mathrm{ml}^{-1}$.

Chemicals and reagents. All reagents were of analytical quality. [carbonyl $\left.-{ }^{14} \mathrm{C}\right] \mathrm{NAD}$ for the production of [carbonyl $\left.{ }^{1+} \mathrm{C}\right]-\mathrm{NMN}$ was purchased from Amersham. NA, NAm, quinolinic acid, NMN and NAD were all purchased from Sigma and were checked for purity using paper chromatography. Ethyl methanesulphonate (EMS) was purchased from Eastman Organic Chemicals. NAmR was prepared as described earlier (Liu et al., 1982).

Procedures. EMS mutagenesis and penicillin enrichment were performed as previously described (Foster $t$ al., $1979 a$ ). The selection of galE mutants used the method outlined by Mojica-A (1975). The transducing phage used was the high transducing derivative of P22, HT105/1-int (Schmieger, 1971). P1 transduction procedures utilized the PI CM clr 100 mutant and the protocol described by Mojica-A (1975). Tn 10 insertions near various loci were isolated as outlined by Chumley et al. (1979). Deletions were isolated based upon $\operatorname{Tn} 10$ excisions as described by Bochner $e t$ al. (1980). Mud(Aplac) directed gene fusions were constructed as described previously (Holley \& Foster, 1982; Hughes \& Roth, 1984). 
Table 1. Strains of Salmonella typhimurium LT-2 used

Strain

\begin{tabular}{|c|c|}
\hline SF24 & $\operatorname{ser} B 54$ \\
\hline SF27 (TT459) & pyrC691:: $\operatorname{Tn} 10$ \\
\hline SF67 & nad $A 540:: \operatorname{Tn} 10$ \\
\hline SF71 (TT6734) & nadA56 pncAI5 zjj-1004:: $\operatorname{Tn} 10 \operatorname{trp} A 49\left(\operatorname{Tn} 10\right.$ near pnuA $\left.A^{+}\right)$ \\
\hline SF77 & nadB5I pnc $A 15$ pnuBI trpA49 \\
\hline SF78 (TR5654) & $\operatorname{thr} A 9 \operatorname{rps} L$ \\
\hline SF81 (TR5657) & purE rps $L$ \\
\hline SF82 (TR5658) & pyrC rpsL \\
\hline SF83 (TR5659) & purB $\operatorname{rps} L$ \\
\hline SF84 (TR5660) & pyrF rpsL \\
\hline SF102 (SGSC183) & $\operatorname{trp} R 520 \operatorname{thrC} 59$ \\
\hline SF103 (SGSC194) & $\operatorname{trp} R 520 \operatorname{ser} B 80$ \\
\hline SF 110 & pyrF146 pde-1 aphA nap gal \\
\hline JF22 & nadB3I purC7 proA46 ilv-405 rha-461 rpsL \\
\hline JF63 & nadB5I pncA $15 \operatorname{trp} A 49$ \\
\hline JF66 & nadA56 pncA15 trpA49 \\
\hline JF76 & nadA56 pncA 15 pnuA11 trpA49 \\
\hline JF85 & nadB31 pnuD2 purC7 proA46 ilv-405 rha-461 rpsL \\
\hline JF181 & nadB31 purC7 proA46 ilv-405 zga-21:: Tn 10 \\
\hline JFI84 & nadA56 pncA15 pnuA11 trpA49 zjj-1004::Tn 10 \\
\hline JF204 & nadB5I zcf-I::Tn $10\left(\operatorname{Tn} 10\right.$ near pnc $\left.A^{+}\right) \operatorname{trp} A 49$ \\
\hline JF211 & nad $A:: \operatorname{Tn} 10$ galE hutR49 $\triangle p n c X 503$ \\
\hline JF339 & gale AnadA $\triangle p n c A$ hut 449 \\
\hline JF349 & nadB5I pncA15 pnuBI pnuCI $\operatorname{trpA49}$ \\
\hline JF350 & nadB5I pncAIS pnuBI pnuC2 trpA49 \\
\hline JF436 & galE pyrC rpsL \\
\hline JF439 & nadB5I nadA:: Tn 10 pnuBI pnuCl trpA49 \\
\hline JF454 & nadB5I pncA 15 pnuBI pnuC3 $\operatorname{trp} A 49$ \\
\hline JF455 & nadB5I pncA15 pnuBI pnuC4 trpA49 \\
\hline JF456 & nadB5I pncA15 pnuBI pnuC5 trpA49 \\
\hline JF457 & nadB5I pncA15 pпuBI pnuC6 trpA49 \\
\hline JF536 & $\operatorname{trpA49}$ nadA56 pncA15 $\triangle p n u A l$ \\
\hline JF572 & $\operatorname{trp} A 49$ nadB5I pncA15 thrA9 zjj-1004::Tn10 \\
\hline JF573 & $\operatorname{trpA49}$ nadB51 pncA15 pnuBl thrA9 zjj-1004::Tn 10 \\
\hline JF666 & nadB5I pncA15 pnuBI pyrC691::Tn 10 \\
\hline JF680 & $\Delta$ nadB pnc $A:: \operatorname{Tn} 10$ pyrFl46 pde-1 aphA nap gal \\
\hline JF681 & nadB51 pncA15 trpA49 pyrC691:: Tn 10 \\
\hline JF682 & nadA56 pncA15 trpA49 pnuA11 pyrC691:: Tn 10 \\
\hline JF731 & pnuC8::Mud1-8 nadB541::Tn 10 supD 10 \\
\hline JF756 & nadB5I pncA15 trpA49 pnuBI pnuC2 rpsL \\
\hline JF806 & $\triangle n a d B$ nad $A 540:: T n 10$ pnuC8::Mud1-8 \\
\hline JF808 & thrA9 pnuC8::Mud1-8 rpsL \\
\hline JF858 & nadB5I pncA15 trpA49 pnuBI pnuC8::Mud1-8 \\
\hline JF869 & pnuC8::Mud1-8 nadRI pyrD95 zff-28::Tn 10 \\
\hline JF870 & nadB5I pncA15 trpA49 pnuC8::Mud1-8 \\
\hline JF871 & $\triangle n a d B$ pnuC8:: Mudl-8 cya::Tn 10 \\
\hline JF873 & nadA56 pncA15 pnuA11 trpA49 pnuC8:: Mud1-8 \\
\hline MU27 & AnadB pnc $A:: \operatorname{Tn} 10 \operatorname{ser} A 13$ rfa-3058 (Hfr $\left.117^{\prime} \mathrm{cw}\right)$ \\
\hline MU28 & $\triangle$ nadB pnc $A:: \operatorname{Tn} 10 \operatorname{ser} A 13$ pur-268 (Hfr $\left.123^{\prime} \mathrm{ccw}\right)$ \\
\hline MU30 & zga-21::Tn 10 ser 113 pur-268 $\left(\operatorname{Tn} 10\right.$ near pnuD $\left.D^{+}\right)\left(\mathrm{Hfr} 123^{\prime} \mathrm{ccw}\right)$ \\
\hline
\end{tabular}

Source or reference*

K. Sanderson

J. Roth

J. Roth

J. Roth

Liu et al. (1982)

J. Roth

J. Roth

J. Roth

J. Roth

J. Roth

K. Sanderson

K. Sanderson

Uerkvitz \& Beck (1981)

Foster et al. (1979a)

Foster et al. (1979a)

Kinney et al. (1979)

* Strains for which no source is given were derived in the work described in this paper. K. Sanderson, Department of Biology, University of Calgary, Calgary, Alberta, Canada T2N 1N4; J. Roth, Department of Biology, University of Utah, Salt Lake City, Utah 84112, USA.

Extracts were prepared and NMN deamidase or NMN glycohydrolase activities determined as described earlier (Foster, 1981). Specific activities are expressed as $\mathrm{nmol} \mathrm{h}^{-1}$ (mg protein) ${ }^{-1}$. Protein was determined by the Lowry method.

The various pnu mutants described in this paper were tested for their ability to transport $\left[{ }^{14} \mathrm{C}\right] \mathrm{NMN}$ and $\left[{ }^{1+} \mathrm{C}\right] \mathrm{NA}$ as described earlier (Kinney et al., 1979). 


\section{RESULTS AND DISCUSSION}

pnuB suppresses the expression of pnuA mutations

To determine whether the PnuB- phenotype, i.e. growth of a nadA pncA pnuB mutant on $10^{-5} \mathrm{M}-\mathrm{NMN}$, could be partially or totally reversed by the introduction of $p n u A$, a strain containing a $\operatorname{Tn} 10$ insertion located near pnuA was constructed. HT transducing phage propagated on strain SF71 ( $\operatorname{Tn} 10$ near $p n u A^{+}$) was used to transduce strain JF76 (nadA pnc A pnuA) to tetracycline resistance $\left(\mathrm{Tc}^{\mathrm{R}}\right)$. The $\mathrm{Tc}^{\mathrm{R}}$ colonies were scored on minimal medium supplemented with NA or $10^{-4} \mathrm{M}-\mathrm{NMN}$. Clones that grew on NA but not on NMN should possess the genotype nadA pnc $A$ pnuA zjj-1004:: Tn 10 and thus contain $\mathrm{Tn} 10$ near pnuA. One such strain (JF184) was cured of HT phage and used to prepare another HT lysate. This lysate was crossed with the pnuB mutant (SF77) and its parent (JF63), selecting for $\mathrm{Tc}^{\mathrm{R}}$. $\mathrm{The} \mathrm{Tc}^{\mathrm{R}}$ transductants were then scored for the PnuA and PnuB phenotypes. The results (Table 2) indicate that when $p n u A$ was transduced into a $n a d B$ pncA strain (JF63) the PnuA- phenotype was observed. The PnuA- phenotype, however, was not observed in the nadB pncA pnuB background. Demonstration that pnuA was successfully transferred to the pnuB strain (SF77) was accomplished by growing phage on 10 of the $\mathrm{Tc}^{\mathrm{R}}$ transductants and subsequently transferring the cryptic pnuA locus to strain JF63. Six of these lysates yielded $\mathrm{Tc}^{\mathrm{R}} p n u A$ progeny. Thus, the $p n u B$ mutation suppresses pnu $A$ mutations. While $p n u B$ seems to map near $p n u A, p n u A$ and $p n u B$ are nevertheless separate loci.

The $p n u B$ suppression of the PnuA- phenotype was not the result of an unusual subunit interaction between the $p n u A$ and $p n u B$ gene products. This was shown by constructing a $\operatorname{Tn} 10$ directed pnuA deletion and cotransducing this deletion into JF572 and JF573, thr $A$ derivatives of JF63 (nadB pncA) and SF77 (nadB pnc $A$ pnuB) respectively. Transductants which were $t h r A^{+}$ were scored for their ability to utilize NMN. As expected, the pnuA deletion phenotype was observed in the JF572 cotransductants ( $\mathrm{NMN}^{-}$) but not in the JF573 cotransductants (NMN ${ }^{+}$). Three-point crosses were performed to determine more accurately the position of $z j j$ $1004:: \operatorname{Tn} 10$ and thus pnuA and pnuB. The results (Fig. 2) show that the Tn 10 insertion mapped between the $\operatorname{ser} B$ and $\operatorname{trp} R$ loci.

\section{Isolation of pnuC mutants}

To define the relationship between $p п u A$ and $p п u B$ more precisely an attempt was made to isolate $p n u B$ reversion mutants or mutants altered in a potential regulatory locus affecting $p n u A$ and/or $p n u B$. SF77 was mutagenized and enriched using penicillin selection to select for mutants which would not grow on $10^{-4} \mathrm{M}-\mathrm{NMN}$ but which could still utilize NA. From approximately 2700 colonies, six were found which were unable to grow on $10^{-4} \mathrm{M}-\mathrm{NMN}$. Two of these were picked for further study. To show that the defect did not alter NAD metabolism at a point following NMN transport, NMN deamidase activity was measured. NMN deamidase activity was normal in both pnuC strains [ $\left.\sim 60 \mathrm{nmol} \mathrm{h}^{-1}(\mathrm{mg} \text { protein) })^{-1}\right]$ indicating they were not NMN deamidase mutants but were probably defective in NMN transport (we show below that they were not defective in NMN glycohydrolase). Accordingly, we designated these strains as pyridine nucleotide uptake mutants, $p n u C$. The biochemical reactions subsequent to NMN deamidase in PNC IV were considered normal since a defect in any one of them would preclude growth on NA as well as on NMN.

Table 2. Transduction of pnuA and pnuB with zjj-1004::Tn10

\begin{tabular}{|c|c|c|c|c|c|}
\hline Donor & Recipient & $\begin{array}{l}\text { Selected } \\
\text { marker }\end{array}$ & $\begin{array}{l}\text { Unselected } \\
\text { marker }\end{array}$ & $\begin{array}{l}\text { Number } \\
\text { scored }\end{array}$ & $\begin{array}{c}\text { Percentage } \\
\text { cotransduction }\end{array}$ \\
\hline SF71 & $\mathrm{JF} 76(\operatorname{nad} A \operatorname{pnc} A$ pnuA $)$ & $T c^{R}$ & pnu $A^{+}$ & 99 & 71 \\
\hline JF 184 & $\mathrm{JF} 63(\operatorname{nad} B \operatorname{pnc} A)$ & $\mathrm{Tc}^{\mathrm{R}}$ & pпuA & 155 & 67 \\
\hline JF 184 & SF77 (nadB pncA pnuB) & $\mathrm{Tc}^{\mathrm{R}}$ & pnuA & 111 & $<1$ \\
\hline SF7! & SF77 (nadB pnc $A$ pnuB) & $T c^{R}$ & pnu $B^{+}$ & 169 & $11 \cdot 5$ \\
\hline
\end{tabular}




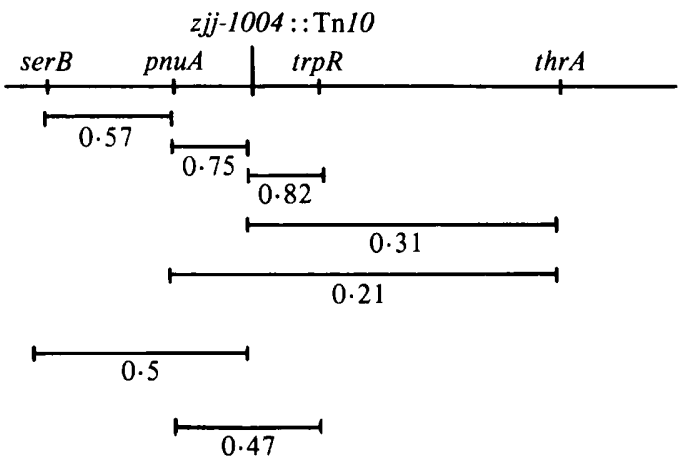

Fig. 2. Location of pnuA in the ser $B$ thr $A$ region of the $S$. typhimurium linkage map. The numbers below the lower bars are the cotransduction frequencies.

Table 3. Transport of $\left[{ }^{14} \mathrm{C}\right] N M N$ and $\left[{ }^{14} \mathrm{C}\right] N A$ by pnu mutants

Cells $\left(2 \times 10^{8} \mathrm{ml}^{-1}\right)$ were suspended in a minimal medium supplemented with $0 \cdot 1 \mu \mathrm{Ci}\left[{ }^{14} \mathrm{C}\right] \mathrm{NA}$ or

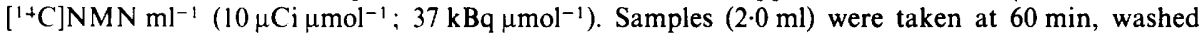
twice rapidly with $5.0 \mathrm{ml}$ cold $\mathrm{E}$ buffer using a Millipore syringe apparatus and the filter suspended and counted directly in $10 \mathrm{ml}$ scintillation fluid.

\begin{tabular}{|c|c|c|c|}
\hline Strain & Pertinent genotype & {$\left[{ }^{14} \mathrm{C}\right] \mathrm{NMN}$} & {$\left[{ }^{14} \mathrm{C}\right] \mathrm{NA}$} \\
\hline $\begin{array}{l}\text { JF66 } \\
\text { JF76 } \\
\text { SF77 }\end{array}$ & 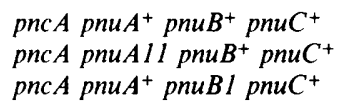 & $\begin{array}{l}0.45 \\
0 \cdot 05 \\
0.79\end{array}$ & $\begin{array}{l}0.83 \\
0.94 \\
0.75\end{array}$ \\
\hline $\begin{array}{l}\text { JF349 } \\
\text { JF350 } \\
\text { JF454 } \\
\text { JF455 } \\
\text { JF456 } \\
\text { JF457 }\end{array}$ & 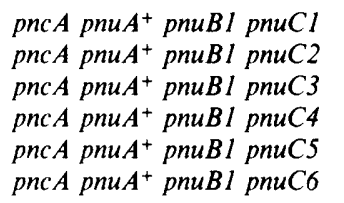 & $\begin{array}{l}0.04 \\
0.07 \\
0.04 \\
0.04 \\
0.06 \\
0.05\end{array}$ & $\begin{array}{l}0.71 \\
0.86 \\
0.81 \\
0.99 \\
0.72 \\
1.01\end{array}$ \\
\hline JF870 & pncA pnu $A^{+}$pnu $B^{+}$pnuC8 & 0.05 & 0.91 \\
\hline
\end{tabular}

pnuC mutants are defective in the transport of $N M N$

Table 3 presents the results of several transport experiments. The pnuA mutant (JF76) was defective in NMN transport while the pnuB mutant (SF77) was able to transport NMN more readily than its parent, as expected. pnuC mutations prevent the transport of NMN in a pnuB background (e.g. JF349) as well as in a pnuB $B^{+}$background (JF870). A model suggested by these results is that the $p n u B$ mutation results in a membrane alteration which facilitates NMN uptake as suggested previously (Liu et al., 1982) but which is independent of the pnuA $A^{+}$system. The $p n u C$ gene product, however, must interact with the $p n u A$ gene product under normal conditions to permit NMN utilization. The pnuB mutation would result in a gene product that, while not requiring the pnuA gene product, still depends upon the presence of the $p n u C$ gene product for NMN uptake.

\section{pnuC is distinct from pnuA and pnuB}

Since two other $p n u$ loci have been identified ( $p n u A$ and $p n u B$ ) it was necessary to determine if $p n u C$ was linked to these loci. The $p n u C$ locus was shown to be different from pnuA by crossing SF71 ( $T$ n 10 near $p n u A^{+}$) with JF349, selecting for $\mathrm{Tc}^{\mathrm{R}}$ and looking for repair of the pnuC mutation. There was no repair of the $p n u C$ locus, indicating that $p n u A$ and $p n u C$ are separate loci (data not shown). 
Table 4. Transduction of pnuC with nadA and galE

Phage P22 was grown on JF211 and this was then crossed with JF349. Initial selection was on LB medium (Miller, 1972) containing tetracycline (selection for $\mathrm{Tc}^{\mathrm{R}}$ ) or minimal $\mathrm{E}$ medium supplemented with $10^{-+} \mathrm{M}-\mathrm{NMN}$ (selection for $p n u \mathrm{C}^{+}$).

$\begin{array}{cccc}\text { Selected marker } & \text { Unselected marker } & \text { Number scored } & \text { Percentage cotransduction } \\ \mathrm{Tc}^{\mathrm{R}}(\text { nad } A) & \text { pnuC } & 174 & 83.9 \\ \text { pnuC } & \text { galE } & 174 & <0.5 \\ \mathrm{Tc}^{\mathrm{R}}(\text { nad } A) & \text { galE } & 174 & 6.9\end{array}$

Table 5. Effect of nicotinic acid concentration on the expression of a pnuC-lacZ fusion in various genetic backgrounds

\begin{tabular}{|c|c|c|c|c|}
\hline \multirow[b]{2}{*}{ Strain } & \multirow[b]{2}{*}{ Genotype } & \multicolumn{2}{|c|}{$\begin{array}{c}\beta \text {-Galactosidase } \\
\text { specific activity (Miller units)* }\end{array}$} & \multirow{2}{*}{$\begin{array}{l}\text { Derepression } \\
\text { (fold) }\end{array}$} \\
\hline & & $10^{-4} \mathrm{M}-\mathrm{NA}$ & $10^{-6} \mathrm{M}-\mathrm{NA}$ & \\
\hline JF731 & nadB $:: \operatorname{Tn} 10$ pnuC8:: Mud $1-8$ & $29 \cdot 6$ & $604 \cdot 5$ & $20 \cdot 4$ \\
\hline JF806 & $\Lambda$ nad $B$ nad $A:: \operatorname{Tn} 10$ pnu $C 8::$ Mud $1-8$ & $36 \cdot 4$ & $520 \cdot 1$ & $14 \cdot 3$ \\
\hline JF808 & pnuC8::Mudl-8 nad + & $36 \cdot 9$ & $64 \cdot 7$ & 1.8 \\
\hline JF858 & nadB pnc $A$ рпи $B$ рпи $C 8::$ Mudl-8 & $29 \cdot 0$ & $690 \cdot 7$ & $23 \cdot 8$ \\
\hline JF870 & nadB pnc A pnuC $8::$ Mudl-8 & $23 \cdot 8$ & $529 \cdot 2$ & $22 \cdot 2$ \\
\hline JF869 & nadRl pnuC8::Mudl-8 & $842 \cdot 1$ & $909 \cdot 3$ & $1 \cdot 1$ \\
\hline JF873 & nadA pncA pnuA pnuC8:: Mudl-8 & $24 \cdot 0$ & $995 \cdot 3$ & $41 \cdot 5$ \\
\hline
\end{tabular}

${ }^{*}$ Miller, 1972. Means of three independent experiments.

The $p n u C$ mutation was also shown to be distinct from the pnuB locus in that $p n u B$ strains repaired for the $p n u C$ mutation were once again able to grow on $10^{-5} \mathrm{M}-\mathrm{NMN}$. Thus, the $p n u B$ phenotype remained even though $p n u C$ was repaired. Consequently, $p n u A, p n u B$ and $p n u C$ are all distinct genetic loci which affect the utilization of NMN.

\section{Repair of pnc A in a pnuC mutant}

To determine if $p n u C$ codes for a transport system totally distinct from the proposed PNC VI NMN glycohydrolase-dependent system (Foster et al., 1979a), the $p n c A^{+}$allele was introduced into JF349 by transduction. If $p n u C$ is distinct from the NMN glycohydrolase system, repair of $p n c A$ should enable the strain to grow on NMN. Alternatively, if pnuC codes for a common transport system then the resultant strain should still be unable to grow on NMN. JF349 was transduced with P22 phage grown on JF204 and 20 transductants showing the pnc $A^{+}$phenotype (growth on NAm) were tested for their ability to grow on $10^{-4} \mathrm{M}-\mathrm{NMN}$. All 20 showed a renewed ability to grow on $10^{-4} \mathrm{M}-\mathrm{NMN}$, indicating that $p n u C$ is distinct from $\mathrm{NMN}$ glycohydrolase-dependent utilization of NMN.

\section{Mapping of the pnuC locus}

The relative location of the pnuC locus on the $S$. typhimurium linkage map was determined by time of entry experiments. Hfr strains MU27 and MU28 were crossed with JF756 with selection for $p n u C^{+}$or $\mathrm{Tc}^{\mathrm{R}}$. The results indicated a general map position for $p n u C$ between 17 and 22 units. Further cotransductional mapping studies with various $\mathrm{Tn} 10 \mathrm{~s}$ distributed throughout this region revealed that $p n u C$ was cotransducible with nadA::Tn 10 . Cotransduction frequencies varied from 83 to $90 \%$ depending upon the $p n u C$ mutant used. Transductional analyses presented in Table 4 indicate the gene order pnuC-nadA-gal.

\section{Expression of pnuC-lacZ gene fusions}

A pnuC:: Mud1-8 $\mathrm{Lac}^{+}$fusion strain (JF731) was isolated by standard Mud(Aplac) phage gene fusion techniques. Measurement of $\beta$-galactosidase activity indicated that $p n u C$ could be regulated by varying the amount of NA supplied to the nadB pnuC::Mudl-8 strain (Table 5). 


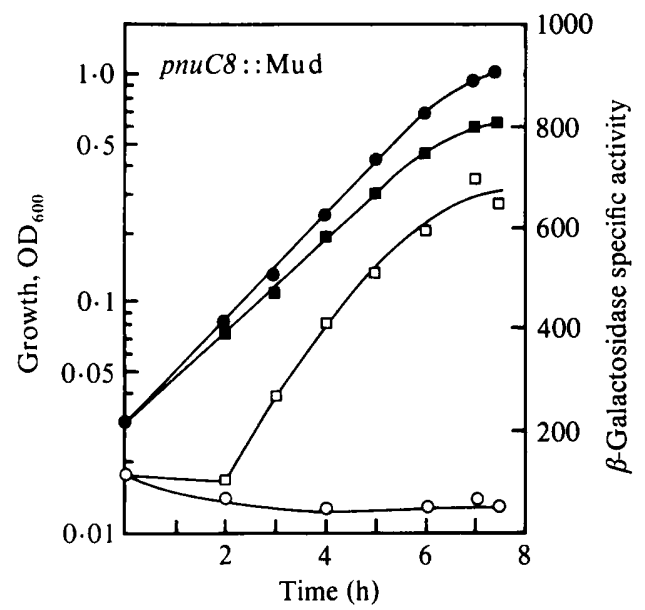

Fig. 3. Kinetics of pnuC derepression. An overnight culture of JF731 was washed twice to remove NA and used to inoculate minimal medium supplemented with either $10^{-4} \mathrm{M}-\mathrm{NA}$ (circles) or $10^{-6} \mathrm{M}-\mathrm{NA}$ (squares). Growth (filled symbols) and $\beta$-galactosidase activity (in Miller units; Miller, 1972) (open symbols) were measured periodically.

Kinetics of pnuC-lac $Z$ derepression during NA starvation are presented in Fig. 3. Derepression occurred midway through starvation. This indicates that $p n u C$ is regulated by fluctuations in intracellular NAD levels as are nadA and $n a d B$ (unpublished observations).

The pnuC::Mud insertion, when transferred to a $\mathrm{nad}^{+}$background, did not result in a $\mathrm{Nad}^{-}$ phenotype. Neither did a nadA : :Tn 10 insertion affect pnuC-lacZ expression (JF806, Table 5). Thus, nad $A$ and pnuC represent two distinct and separate loci.

\section{Regulation of pnuC by nadR}

A mutation which results in the constitutive expression of $\operatorname{nadA}$ and $\operatorname{nad} B$ was recently isolated in a gene designated $n a d R$ (unpublished observations). To determine whether $n a d R$ also affects pnuC regulation, pnuC8:: Mudl-8 was transferred to the nadR strain (JF869) and $\beta$ galactosidase activity monitored during growth in high and low concentrations of NA. The results (Table 5) reveal a high constitutive expression of pnuC in the $n a d R$ background indicating a role for $n a d R$ in the regulation of pnuC.

The effect of pnuB or pnuA mutations on $p n u C-l a c Z$ expression was examined. While $p n u B$ did not affect $p n u C-l a c Z$ expression (JF858), the pnuA mutation tested (JF873) allowed greater derepression of $p n u C$ indicating that $p n u A$ as well as nadR may act as a negative effector of $p n u C$ (Table 5).

\section{Isolation and mapping of a pnuD mutant affected in PNC VI-dependent NMN transport}

To reveal potential genetic determinants involved in the PNC VI-dependent transport of NMN, a search was made for mutations in a nad pnc $A^{+}$strain (JF22) which would prevent growth on $10^{-5} \mathrm{M}-\mathrm{NMN}$. Again, EMS mutagenesis was used with penicillin enrichment. One of the mutants isolated (JF85) grew well on $10^{-5} \mathrm{M}-\mathrm{NAm}$ and $10^{-4} \mathrm{M}-\mathrm{NMN}$ but failed to grow on $10^{-5} \mathrm{M}-\mathrm{NMN}$, in contrast to the parent, JF22, which grew well on $10^{-5} \mathrm{M}-\mathrm{NMN}$. Extracts of the pnuD mutant were compared with those of JF22 to determine the relative activities of NMN glycohydrolase and NMN deamidase. Both enzymes appeared normal in JF85 (data not shown).

Tn 10 insertions were isolated near the pnuD locus by selecting for $p n u D^{+}\left(\mathrm{NMN}^{+}\right) \mathrm{Tc}^{\mathrm{R}}$ strains following the transduction of JF85 with a P22HT lysate propagated on a pool of $\operatorname{Tn} 10$ insertion mutants. The Tn10 insertion isolated (JF181) was transferred to the Hfr strains SA722 and SA464 and the resultant $\mathrm{Tn} 10$ derivations used to transfer $T \mathrm{c}^{\mathrm{R}}$ in interrupted matings to suitable auxotrophic mutants. The time of entry experiments indicate a map position for $p n u D$ of 56-60 units on the $S$. typhimurium linkage map. 
Table 6. Growth characteristics of pnuC in various genetic backgrounds

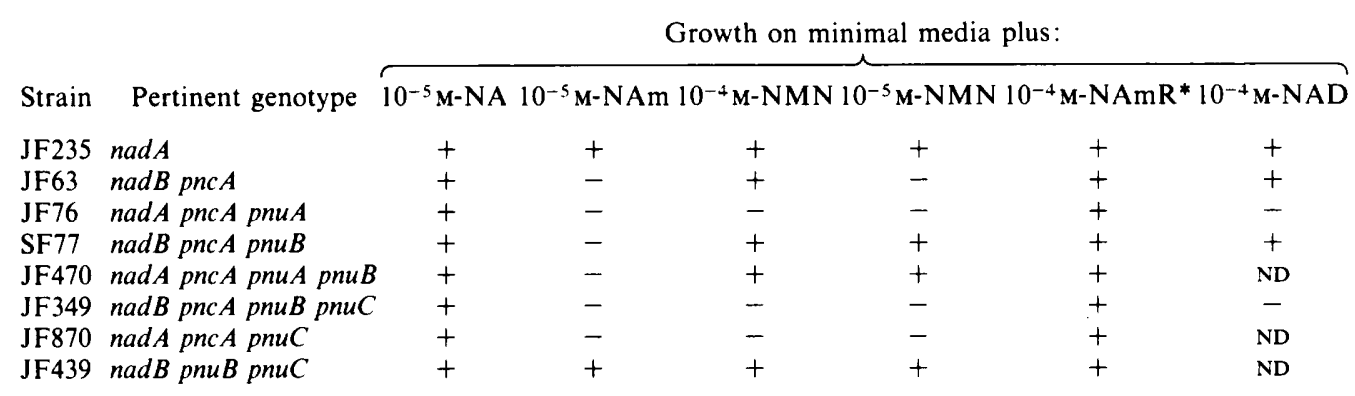

+ , Growth; - , no growth; ND, not determined.

* Growth of strain JF76 on NAmR was less than that of the other strains tested.

Table 7. Effect of pnu and phosphatase mutations on the utilization of NMN and UMP

NA and NAm were used at a concentration of $10^{-5} \mathrm{M}$, and uracil at $2.5 \times 10^{-4} \mathrm{M}$.

\begin{tabular}{|c|c|c|c|c|c|c|c|c|}
\hline \multirow[b]{2}{*}{ Strain } & \multirow[b]{2}{*}{ Pertinent genotype } & \multicolumn{7}{|c|}{ Growth on minimal medium plus: } \\
\hline & & Ura & $\begin{array}{c}\text { Ura } \\
+ \text { NA }\end{array}$ & $\begin{array}{c}\text { Ura } \\
+ \text { NAm }\end{array}$ & $\begin{array}{c}\text { Ura } \\
+10^{-4} \mathrm{M}- \\
\text { NMN }\end{array}$ & $\begin{array}{c}\text { Ura } \\
+10^{-5} \mathrm{M}^{-} \\
\mathrm{NMN}\end{array}$ & $\begin{array}{c}10^{-3} \mathrm{M}^{-} \\
\text {UMP } \\
+ \text { NA }\end{array}$ & $\begin{array}{c}10^{-4} \mathrm{M}^{-} \\
\text {UMP } \\
+\mathrm{NA}\end{array}$ \\
\hline SF27 & pyrC & + & ND & ND & ND & ND & + & - \\
\hline JF681 & nadB pncA pnu $A^{+}$pyrC & - & + & - & + & - & + & - \\
\hline JF682 & nadB pncA pnuA pyrC & - & + & - & - & - & + & - \\
\hline JF666 & nadB pncA pnuB pyrC & - & + & - & + & + & + & - \\
\hline JF680 & nadB pncA pyrF aphA nap pde & - & + & - & + & - & - & - \\
\hline
\end{tabular}

+ , Growth; -, no growth; ND, not determined.

\section{Genetic relationships between pnu loci}

Further insight into the relationships between pnuC and the other pnu loci was afforded by introducing $p n u C$ by transduction into strains with various genetic backgrounds. These were then tested on minimal media supplemented with various components of the PNC. Although $p n u C$ was identified in a $p n u B$ mutant the $p n u C$ phenotype does not require a $p n u B$ background to prevent growth on NMN (Table 6). This can be seen by comparing the growth patterns of JF349

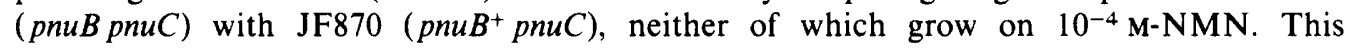
information complements the transport studies presented above, as does the observation that the pnuB mutation suppresses the expression of pnuA mutations. This is seen by comparing JF76 ( $p n u A$ ), which did not grow on NMN supplemented medium, to JF470 (pnuA pnuB), which grew well on this medium.

The pnu system is not associated with nonspecific phosphatases

The fact that phoP, a gene involved in nonspecific acid phosphatase regulation, is also found in this region led to the idea that $p n u C$ may be the phoP locus (Kier et al., 1979). Both purine and pyrimidine nucleotides are acted upon by nonspecific acid phosphatases to yield their respective nucleosides which may then enter the cell. Since NMN is structurally similar to pyrimidine nucleotides it was reasonable to suppose that NMN might be utilized through the same reaction. However, the lack of $p n u C$ linkage to $p u r B$ contradicts this since $p h o P$ is $96 \%$ linked to $p u r B$ by P22 phage transduction (Kier et al., 1979). 
To determine more directly the possible involvement of $p n u$ loci with the transport of other nucleotides, nadB and $p n c A$ were introduced into SF110, a pde aphA nap mutant lacking nonspecific acid phosphatase I (nap), nonspecific acid phosphatase II (aphA) and 2': $3^{\prime}$-cyclicnucleotide 2'-phosphodiesterase ( $p$ de). S. typhimurium strains lacking these phosphatases fail to degrade nucleoside monophosphates such as UMP and thus cannot utilize nucleotides to satisfy auxotrophic requirements (Uerkvitz \& Beck, 1981). If the $p n u A^{+} p n u B^{+}$system for $\mathrm{NMN}$ utilization is related to these nucleotide phosphatases, then the nadB pncA pde aphA nap strain (JF680) should be unable to grow on $10^{-4} \mathrm{M}-\mathrm{NMN}$. This strain, however, did grow normally on this concentration of $\mathrm{NMN}$, indicating that $p n u A^{+} p n u B^{+}$dependent transport is unrelated to systems used to degrade and transport UMP (Table 7). Further evidence for the specificity of NMN utilization is provided by JF682, a pnuA pyrC mutant, which grew normally on $10^{-3} \mathbf{M}^{-}$ UMP but failed to grow on NMN. The data in Table 7 also reveal that the pnuB pyrC strain, JF666, while it could utilize lower concentrations of NMN did not grow any better on lower UMP levels. Thus, NMN is not utilized by systems involved in the transport of other structurally similar nucleotides.

In summary, the genetic and biochemical data suggest that under normal conditions the $p n u A^{+}$and $p n u C^{+}$gene products form a system for NMN uptake. The $p n u B^{+}$gene product plays no role in this system. Selective pressure for a more efficient transport system, however, appears to result in an alteration of $p n u B$ allowing it to interact either directly or indirectly with the $p n u C^{+}$gene product to transport NMN more efficiently. In this sytem $p n u A^{+}$plays no role. None of the above loci appeared to function in the utilization of NMN via the PNC VI/pnc $A$ route, but a fourth locus, pnuD, was found to be involved in this system.

It is interesting that both pnuA and pnuC mutants are capable of growing on NAmR. This suggests that the $p n u$ system is not involved with NAmR uptake and that a separate uptake system is required for this nucleoside. This is supported by the recent finding of a NAmR salvage pathway in $S$. typhimurium (Liu et al., 1982).

We thank A. G. Moat for his expert artwork and B. M. Olivera, J. Roth and K. Hughes for their helpful discussions.

This work was supported by Public Health Service grants 1 R01 GM-32595 and 1 S07 RR05870-01 from the National Institutes of Health.

\section{REFERENCES}

Bochner, B. R., HuANG, H., SChieven, G. L. \& AmES, B. N. (1980). Positive selection for loss of tetracycline resistance. Journal of Bacteriology 143, 926-933.

Chumley, F. G., Menzel, R. \& Roth, J. R. (1979). Hfr formation directed by Tnl0. Genetics 91, 639655.

Foster, J. W. (1981). Pyridine nucleotide cycle of Salmonella typhimurium: in vitro demonstration of nicotinamide adenine dinucleotide glycohydrolase, nicotinamide mononucleotide glycohydrolase and nicotinamide adenine dinucleotide pyrophosphatase activities. Journal of Bacteriology 145, 1002-1009.

Foster, J. W. \& Baskowsky-Foster, A. M. (1980). Pyridine nucleotide cycle of Salmonella typhimurium: in vivo recycling of nicotinamide adenine dinucleotide. Journal of Bacteriology 142, 1032-1035.

Foster, J. W. \& MoAT, A. G. (1980). Nicotinamide adenine dinucleotide biosynthesis and pyridine nucleotide cycle metabolism in microbial systems. Microbiological Reviews 44, 83-105.

FOSTER, J. W., KINNEY, D. M. \& MOAT, A. G. (1979a). Pyridine nucleotide cycle of Salmonella typhimurium: isolation and characterization of $p n c A, p n c B$ and pnc $C$ mutants and utilization of exogenous nicotinamide adenine dinucleotide. Journal of Bacteriology 137, 1165-1175.
Foster, J. W., Kinney, D. M. \& Moat, A. G. (1979b). Pyridine nucleotide cycle of Salmonella typhimurium: regulation of nicotinic acid phosphoribosyltransferase and nicotinamide deamidase. Journal of Bacteriology 138, 957-961.

Holley, E. A. \& Foster, J. (1982). Bacteriophage P22 as a vector for $\mathrm{Mu}$ mutagenesis in Salmonella typhimurium: isolation of nad-lac gene fusions. Journal of Bacteriology 152, 959-962.

Hughes, K. T. \& RoTH, J. R. (1984). Conditionally transposition-defective derivative of Mudl(amp lac). Journal of Bacteriology 159, 130-137.

KieR, L. D., Weppelman, R. M. \& Ames, B. N. (1979). Regulation of nonspecific acid phosphatase in Salmonella: phoN and phoP genes. Journal of Bacteriology 138, 155-161.

Kinney, D. M., Foster, J. W. \& Moat, A. G. (1979). Pyridine nucleotide cycle of Salmonella typhimurium: in vitro demonstration of nicotinamide mononucleotide deamidase and characterization of pnuA mutants defective in nicotinamide mononucleotide transport. Journal of Bacteriology 140, 607-611.

LiU, B. P., Foster, J. W., Manalapaz-Ramos, P. \& OlIVERA, B. M. (1982). A nucleoside salvage pathway for NAD biosynthesis in Salmonella typhimurium. Journal of Bacteriology 152, 1111-1116. 
MILleR, J. H. (1972). Experiments in Molecular Genetics. Cold Spring Harbor, NY: Cold Spring Harbor Laboratory.

Mojica-A, T. (1975). Transduction by phage P1 Cm clr 100 in Salmonella typhimurium. Molecular and General Genetics 138, 113-126.

SCHMIEGER, H. (1971). A method for detection of phage mutants with altered transducing ability. Molecular and General Genetics 110, 378-381.
Uerkvitz, W. \& BeCK, C. F. (1981). Periplasmic phosphatases in Salmonella typhimurium LT2. A biochemical, physiological and partial genetic analysis of three nucleoside monophosphate dephosphorylating enzymes. Journal of Biological Chemistry 256, 382-389.

VoGel, H. J. \& BonNeR, D. M. (1956). Acetylornithase of Escherichia coli: partial purification and some properties. Journal of Biological Chemistry 93, 237244. 